\title{
Antifungal activity of Rauwolfia serpentina root extract against Neurospora crassa and genetic study of induced mutants
}

\author{
A. L. Ray \\ Department of Botany, Jagannath University, Dhaka-1100, Bangladesh
}

\begin{abstract}
\section{Introduction}

The pink bread mold Neurospora crassa is a model organism that play a role in developing molecular and cell biology. $N$. crassa has been used intensively in many laboratories since 1941 (Perkins, 2000). Life cycle of this haploid organism is short and zygote is only diploid. For genetic study, many mutant strains and stocks of $N$. crassa are available and it is widely distributed throughout the world. Beadle and Tatum exposed x-rays to $N$. crassa causing mutations and won Nobel Prize in 1958 by establishing "one gene, one enzyme" hypothesis.
\end{abstract}

Rauwolfia serpentina ( $R$. serpentina) root extract was used to study antifungal activity against Neurospora crassa (Ema) as well as mutation, mating type, segregation of the fungus. It was found that more concentration of root extract was used in VM media, higher antifungal activity was observed. After mutation in $N$. crassa conidia with root extract of $R$. serpentina, 5 groups of morphological mutants viz. albino (al 98), vigorous ( $v g$ 77), fluffy (fl 139), conidial band (con.band 221) and cauliflower (clf 155) were obtained. From genetic study, it was found that all the mutants formed perithecia crossing with EmA which confirmed that mating type of all the mutants is 'a' and is not changed due to mutation. Besides, segregation ratio between mutant and wild isolates was about 1:1 which confirmed that mutation takes place in the nuclear gene, not cytoplasmic.

Keywords: Neurospora crassa; Antifungal activity; Mutation; Mating type; Segregation
As like physical mutagen, researchers worked with plant extract that possess antifungal property (Haque and Shamsi, 1996 \& 1997; Kumari et al., 2016) and successfully used for the study of genetics of $N$. crassa as well (Mozmader et al., 2000, 2002; Ray and Rahim, 2010; Ray et al., 2010). $R$. serpentina is a medicinal plant and its root contains 50 indole alkaloids as well as rutin, oleoresin, sterol, oleic acid and unsaturated alcohols (Ghani, 2003). The alkaloids are the sources of chemical mutagens that inhibit the growth of microorganism and thus successfully be used for induction of mutation to study some genetic parameter.
Study of mating type, fertility and segregation are very important parameters to investigate genetics of induced mutants. The necessity of studying these parameters is to know whether any change in Mendelian ratio is caused due to mutation.

In this context, the present research was undertaken to study - a) antifungal activity of root extract of $R$. serpentina against $N$. crassa, b) induction of mutation in $N$. crassa, and c) study of genetic variability of mating type and segregation of induced mutants.

\section{Materials and methods}

N. crassa (culture Ema 5297 and EmA 5296) was the experimental material and obtained from Microbial Genetics Stock of Department of Botany, University of Dhaka. $R$. serpentina root extract was obtained from the garden of Department of Botany of University of Dhaka and was used for the study of antifungal activity and induction of mutation in $N$. crassa. To obtain different media, various stock solutions were prepared. Vogel's minimal medium, VM (Vogel, 1956) was used for culturing N. crassa in the test tube and counting the spores on plate. Westergaard Crossing Medum, WCM (Westergaard and Mitchel, 1947) was used for crossing. It restricts vegetative growth but enhance sexual 
reproduction. Sorbose minimal medium (SM) was used for plating. It also restricts growth so that colonies do not run into each other which could happen in VM.

\section{Study of antifungal activity}

To study antifungal activity of $R$. serpentina root extract against $N$. crassa (Ema), different concentrations of root extract were prepared and the effect was evaluated by measuring the radial growth of mycelia of Ema on VM agar plates.

Preparation of different concentration of $R$. serpentina root extract

To prepare different concentration of $R$. serpentina root extract, $100 \mathrm{gm}$ root was taken and washed 2 times with tap water and 2 times with distilled water. After air drying the root was cutted into small pieces, added $5 \mathrm{ml}$ of sterilized distilled water and grinded in an electric grinder. The paste was filtered with a sterilized cotton cloth to get clear solution. Then the solution was taken in a centrifuge tube and centrifuged with a centrifuge machine for 5 minutes at 3000 rpm. It was followed 3 times and at every stage the upper part of the solution (supernatant) was taken in another centrifuge tube to remove the lower part (debris). This amount $(5 \mathrm{ml})$ is considered as the $100 \%$ concentration of root extract.

Effect of root extract of $R$. serpentina on the growth of $N$. crassa

Different concentrations of root extract of $R$. serpentina were taken separately on the Petri dish at the rate of $0.5 \mathrm{ml}, 1.0 \mathrm{ml}$, $2.0 \mathrm{ml}, 4.0 \mathrm{ml}$ and only $10 \mathrm{ml}$ of VM (control) on a Petri dish. $10 \mathrm{ml}$ of molten VM was added in each Petri dish and rotated gently for uniform mixing of solution with medium. When the medium became solid, the centre of the Petri dish was marked and fresh culture of Ema was inoculated at that point with a sterilized needle. All the Petri dishes were kept in incubator at $25^{\circ} \mathrm{C}$. After 21, 24, 27 and 30 hours, the radial growth of Ema of each Petri dish was measured in $\mathrm{cm}$.

\section{Induction of mutation}

Mutation in N. crassa was conducted following early reported procedure (Mozmader et al., 2002). For induction of mutation in $N$. crassa (Ema) $4 \mathrm{ml}$ of $R$. serpentina root extract was taken into a centrifuge tube. 1 loop of conidia of Ema was taken into the tube and was shaken for homogenous solution. The tube was kept for 2 hours to occur mutation. After centrifugation, the solution above conidia was poured out from the centrifuge tube. $4 \mathrm{ml}$ of sterilized distill water was added in the centrifuge tube and centrifuged for 5 minutes. Then the distilled water was poured out. The same procedure was repeated 3 times. Then $1 \mathrm{ml}$ of distilled water was added with the treated conidia remaining at the bottom of the centrifuge tube and the tube was shaken well. The sterilized Petri dishes were marked and 1 drop of the suspension was taken accordingly. $10 \mathrm{ml}$ of molten SM media was added to each of the Petri dish and were shaken gently to mix the suspension and media. The plates were kept inside the incubator at $25^{\circ} \mathrm{C}$ for 24 hours for growth of conidia. A number of well separated colonies were isolated from each Petri dish by cutting agar blocks from the conidial colony with an arrow shaped isolating needle and were inoculated into small tubes containing VM media. After 5 days, all the cultures were observed and classified by comparing their characters with wild type Ema. The conidial cultures which had any morphological variations were subcultured several times in small tubes and checked carefully whether any permanent morphological change has taken place.

\section{Determination of mating type}

The previous procedure (Rahim 2001) was followed for determination of mating type of $N$. crassa. To do this Ema, EmA and the mutants were subcultured 3 times on VM media with an interval of 5 days. The mating type of the mutants was determined by crossing them with both Ema and EmA. The result of the crosses was observed for 5-30 days. If perithecia are formed with Ema, the mating type of the mutant is ' $\mathrm{A}$ ' and if perithecia are formed with EmA, mating type is ' $a$ '.

\section{Study of segregation}

Segregation was studied according to Rahim (2001) when the cross between mutants and wild (EmA) formed perithecia and shedded spores. Spores were then spreaded on SM media. In the next step, single isolated spores were inoculated in culturing tubes containing VM media and incubated at $25^{\circ} \mathrm{C}$ for 5 days. The isolates were distinguished as wild and mutant. The ratio between mutant isolates and wild type isolates were calculated.

\section{Results and discussion}

For the study of antifungal activity of $R$. serpentina root extract on the growth of $N$. crassa, different concentrations of $R$. serpentina root extract were prepared according to the Table I.

Root extract of $R$. serpentina showed significant growth inhibitory effect on $N$. crassa (Ema). The effect was determined by measuring the radial growth of mycelia of 
Table I. Different concentrations of Rauwolfia serpentina root extract

\begin{tabular}{ccccc}
\hline $\begin{array}{c}\text { Amount of } \\
\text { root extract } \\
(\mathrm{ml})\end{array}$ & $\begin{array}{c}\text { Amount of } \\
\text { Distilled } \\
\text { water }(\mathrm{ml})\end{array}$ & $\begin{array}{c}\text { Total amount of } \\
\text { solution }(\mathrm{ml})\end{array}$ & $\begin{array}{c}\text { Concentration of } \\
\text { the solution taken } \\
(\mathrm{ml}) /(\%)\end{array}$ & $\begin{array}{c}\text { Percentage of } \\
\text { the root extract } \\
\text { in the solution }\end{array}$ \\
\hline & & $0.5(5 \%)$ & 2.5 \\
5 & 10 & $1.0(10 \%)$ & 5 \\
& & $2.0(20 \%)$ & 10 \\
& & $4.0(40 \%)$ & 20 \\
\hline
\end{tabular}

Table II. Radial growth of Ema on VM agar plates containing root extract of Rauwolfia serpentina

\begin{tabular}{lcccc}
\hline $\begin{array}{c}\text { Concentration of } \\
\text { the solution } \\
(\mathrm{ml}) /(\%)\end{array}$ & \multicolumn{3}{l}{ Radial mycelia growth (in $\mathrm{cm})$ after different time intervals } \\
\cline { 2 - 4 } $0(\mathrm{Control})$ & 2.40 & $(24 \mathrm{~h})$ & $(27 \mathrm{~h})$ & $(30 \mathrm{~h})$ \\
$0.5(5 \%)$ & 2.90 & 3.30 & 3.80 \\
$1.0(10 \%)$ & 0.85 & 1.10 & 1.30 & 1.60 \\
$2.0(20 \%)$ & 0.70 & 0.90 & 1.10 & 1.35 \\
$4.0(40 \%)$ & 0.30 & 0.35 & 0.40 & 0.55 \\
\hline
\end{tabular}

Table III. Classification and Nomenclature of Rauwolfia serpentina root extract induced mutants of Neurospora crassa

\begin{tabular}{ccccc}
\hline GroupName of the mutant & Characteristics & No of & Frequency (\%) \\
\hline A & albino & Both conidia and mycelia are colourless & 28 & 43.08 \\
B & vigorous & Profuse conidial growth touches the plug & 17 & 26.15 \\
C & fluffy & Profuse mycelial growth & 12 & 18.46 \\
D & $\begin{array}{c}\text { conidial } \\
\text { band }\end{array}$ & $\begin{array}{l}\text { Conidia formed a nice band at the tip of } \\
\text { the growth }\end{array}$ & 5 & 7.69 \\
E cauliflower & $\begin{array}{l}\text { Checked growth, conidia form } \\
\text { cauliflower like structure }\end{array}$ & 3 & 4.62 \\
\hline
\end{tabular}


Ema. Lower radial growth indicated higher antifungal activity of $R$. serpentina root extract against $N$. crassa. It was found that more amount of root extract was used in VM media, higher antifungal activity was observed. The finding of this research is also confirmed by the early reported study (Ray et. al., 2010). The result is presented in Table II.

Radial growth of mycelia of Ema was decreased with the increase of $R$. serpentina root extract in the solution. $20 \%$ concentration of solution i.e. $10 \%$ of root extract (Table I) also indicates higher mutagenic effect and used for induction of mutation in N. crassa. with the previous research (Ray and Rahim 2010). From these findings it may be inferred that plant extract is suitable mutagen to obtain morphological mutants, not biochemical.

For determination of mating type, the mutants were crossed with both the two wild opposite mating types (Ema \& EmA). It was observed that all the mutants formed perithecia by the cross with EmA (Table IV). So it is clear that mating type of all the mutants is ' $a$ ' and is not changed due to mutation. This result is also similar as the result obtained earlier (Rahim, 2001).

Table IV. Mating type of Neurospora crassa mutants

\begin{tabular}{|c|c|c|c|c|c|c|c|c|}
\hline $\begin{array}{l}\text { Sl. } \\
\text { no. }\end{array}$ & Designation of the cross & $\begin{array}{l}\text { Whether } \\
\text { perithecia } \\
\text { formed } \\
\text { (Yes/No) }\end{array}$ & $\begin{array}{l}\text { Days of } \\
\text { initiation of } \\
\text { perithecia }\end{array}$ & $\begin{array}{l}\text { Frequency of } \\
\text { perithecia }\end{array}$ & $\begin{array}{l}\text { size of } \\
\text { perithecia }\end{array}$ & $\begin{array}{l}\text { Spore } \\
\text { shedding } \\
\text { days }\end{array}$ & Fertility & $\begin{array}{l}\text { Mating } \\
\text { type }\end{array}$ \\
\hline \multirow[t]{2}{*}{1} & al $98 \mathrm{a} \times \mathrm{EmA}$ & Yes & 15 & Few & Small & 24 & Fertile & $\mathrm{a}$ \\
\hline & al $98 \mathrm{a} \times \mathrm{Ema}$ & No & & & & & & \\
\hline \multirow[t]{2}{*}{2} & $v g 77 \mathrm{a} \times \mathrm{EmA}$ & Yes & 12 & Many & Medium & 19 & Fertile & $\mathrm{a}$ \\
\hline & $v g 77 \mathrm{a} \times \mathrm{Ema}$ & No & & & & & & \\
\hline \multirow[t]{2}{*}{3} & $f l 139 \mathrm{a} \times \mathrm{EmA}$ & Yes & 12 & Many & Large & 18 & Fertile & $\mathrm{a}$ \\
\hline & $f l 139 \mathrm{a} \times \mathrm{Ema}$ & No & & & & & & \\
\hline \multirow[t]{2}{*}{4} & clf $155 \mathrm{a} \times \mathrm{EmA}$ & Yes & 13 & Many & Medium & 19 & Fertile & $\mathrm{a}$ \\
\hline & clf $155 \mathrm{a} \times \mathrm{Ema}$ & No & & & & & & \\
\hline \multirow[t]{2}{*}{5} & con.band $221 \mathrm{a} \times \mathrm{EmA}$ & Yes & 14 & Few & Small & 22 & Fertile & $\mathrm{a}$ \\
\hline & con.band $221 \mathrm{a} \times \mathrm{Ema}$ & No & & & & & & \\
\hline
\end{tabular}

Following the procedure of mutation in N. crassa (Ema, 5297 ) conidia with $10 \%$ root extract of $R$. serpentina, 5 groups of morphological mutants (Table III) were obtained. These are albino (al 98), vigorous (vg 77), fluffy (fl 139), conidial band (con.band 221) and cauliflower (clf 155). From a total of 65 mutants, cauliflower showed the lowest frequency $(4.62 \%)$ and albino showed the highest frequency $(43.08 \%)$. In this experiment no biochemical mutant was obtained and it showed similarity
These all mutants of $N$. crassa induced with root extract of $R$. serpentina were also used for the study of segregation. Segregation was studied by analyzing the ratio between mutant isolates and wild type isolates (Table V).

From the table it is clear that segregation ratio between wild type and mutant isolates was about $1: 1$. So it may be concluded that the mutants obtained are due to the mutation in nuclear gene, not cytoplasmic. 
Table V. Segregation ratio of mutants of Neurospora crassa

\begin{tabular}{|c|c|c|c|c|c|}
\hline no. & Name of the cross & $\begin{array}{l}\text { Total no. of } \\
\text { spore } \\
\text { isolated }\end{array}$ & $\begin{array}{l}\text { No. of wild } \\
\text { isolates }\end{array}$ & $\begin{array}{l}\text { No. of } \\
\text { mutant } \\
\text { isolates }\end{array}$ & $\begin{array}{l}\text { Segregation ratio } \\
\text { (Mutant : Wild) }\end{array}$ \\
\hline 1 & al $98 \mathrm{a} \times \mathrm{EmA}$ & 73 & 39 & 34 & $1: 1.15$ \\
\hline 2 & $v g 77 \mathrm{a} \times \mathrm{EmA}$ & 81 & 37 & 44 & $1: 0.84$ \\
\hline 3 & $f l 139 \mathrm{a} \times \mathrm{EmA}$ & 80 & 44 & 36 & $1: 1.22$ \\
\hline 4 & clf $155 \mathrm{a} \times \mathrm{EmA}$ & 63 & 33 & 30 & $1: 1.1$ \\
\hline 5 & con.band $221 \mathrm{a} \times \mathrm{EmA}$ & 69 & 38 & 31 & $1: 1.23$ \\
\hline
\end{tabular}

\section{Conclusion}

R. serpentina root contains various types of chemicals which restrict the growth of $N$. crassa and thus used as chemical mutagen to induce mutation. It was observed that root extract of $R$. serpentina showed significant growth inhibitory antifungal activity against $N$. crassa (Ema) and the concentration of root extract was $10 \%$ which is suitable for induction of mutation. The study also indicated that chemical mutagen such as root extract of $R$. serpentina is only suitable to obtain morphological mutants. Moreover the findings revealed that mutation has no effect regarding the change of mating type. Besides segregation ratio confirmed that mutation takes place only in the nuclear gene and has no effect on cytoplasmic gene.

Conflict of interest: The author has declared no conflict of interest.

\section{References}

Ghani A (2003), Medicinal plants of Bangladesh, $2^{\text {nd }}$ Ed., Asiatic Society of Bangladesh, Dhaka, pp 364-365.

Haque T and Shamsi S (1996), Activity of certain plant extract against jute stem rot fungus Macrophomina phaseolina,Dhaka Univ J Biol Sci. 5(1): 103 -104.

Haque T and Shamsi S (1997), Activity of some plant extracts on growth of sheth rot pathogen Sarocladium oryzae (Sawada), Rames W and Hawksa D, J. Asiat Soc Bangladesh Sci. 231: 153- 155 .

Kumari S, Siddique FMS, Gupta SD and Baruah A (2016), Antimicrobial property of different parts of Citrus limon, IOSR Journal of Biotechnology and Biochemistry. 2(2): 53-55. DOI:org/10.1186/ 1472-6882-6-39

Mozmader TIMA, Keya US and Haque T (2002), Induction of mutation in Neurospora crassa with aqueous, petroleum ether and chloroform extracts of Neem (Azadirachta Indica A. Juss) leaves, Dhaka Univ J Biol Sci. 11(2): 167 -173.

Mozmader TIMA, Yesmin N and Jabbar A (2000), Induction of mutation in Neurospora crassa with aqueous extract of garlic (Allium sativum L.) and genetical and chromatographic studies of some selected mutants, Dhaka Univ J Biol Sci. 9(2): $143-150$.

Perkins DD and Davis RH (2000), Evidence for safety of Neurospora species for academic and commercial uses, Applied and Environmental Microbiology. 66(12): 5107-5109. DOI: 10.1128/AEM.66. $12.5107-5109.2000$ 
Rahim T (2001), Genetical and biochemical investigations and protoplast fusion of anthranilic acidmutants of Neurospora crassa, Ph.D. Thesis. Department of Botany, University of Dhaka, Dhaka, Bangladesh.

Ray AL and Rahim T (2010), Cellulase and xylanase activities of mutants of Neurospora crassa induced with leaf extract of Abroma augusta L, Bangladesh $J \quad$ Sci Ind Res. 45(2): 151-154. DOI: .org/10.3329/bjsir.v45i2.5713
Ray AL, Jahan MA and Rahim T (2010), Mutation in Neurospora crassa with leaf extract of Citrus aurantifolia and their soluble protein content, Dhaka Univ J Biol Sci. 19(2): 151-155.

Vogel HJ (1956), A convenient growth medium for Neurospora crassa, Microb. Genet. Bull. 13: 42- 43.

Westergaard M and Mitchel HK (1947), Neurospora crassa, Synthetic medium for favouring sexual reproduction, Am. J. Bot. 34: $573-577$. DOI:org/10.1002/j.1537-2197.1947.tb13032.x 